\title{
Monolithically Integratable Colliding Pulse Modelocked Laser Source for O-CDMA Photonic Chip Development
}

\author{
Nicolas K. Fontaine ${ }^{1}$, Jong-Hwa Baek ${ }^{1}$, Chen $\mathrm{Ji}^{1}{ }^{1}$, Ronald G. Broeke ${ }^{1}$, Xiaoping $\mathrm{Zhou}^{1}{ }^{\text {, Sang-Woo Seo }}{ }^{1}$, \\ F. M. Soares ${ }^{1}$, M. Shearn ${ }^{2}$, A. Scherer ${ }^{2}$, F. Olsson ${ }^{3}$, S. Lourdudoss ${ }^{3}$, K. Y. Liu, W. T. Tsang ${ }^{4}$ and S. J. B. Yoo ${ }^{1}$ \\ ${ }^{1}$ Department of Electrical and Computer Engineering, University of California, Davis, 95616 \\ ${ }^{2}$ Division of Engineering and Applied Science, California Institute of Technology, Pasadena, CA 91125 \\ ${ }^{3}$ School of Information and Communication Technology, Royal Institute of Technology, S-16440 Stockholm, Sweden \\ ${ }^{4}$ Multiplex, Inc., 5000 Hadley Road, South Plainfield, New Jersey 07080, USA \\ email:sbyoo@ucdavis.edu
}

\begin{abstract}
We demonstrate modelocking of a colliding-pulse mode-locked laser formed by 3- $\mu$ m-deep etched-mirrors on an InP platform for integration with passive waveguide components. Timing jitter of $243 \mathrm{fs}$ and pulse width of $10 \mathrm{ps}$ were measured.

(C)2008 Optical Society of America

OCIS codes: (060.4250) Networks; (060.4510) Optical communication.
\end{abstract}

\section{Introduction}

Optical code division multiple access (O-CDMA) technology is a promising candidate for the next generation local access networks, with numerous field trials[1] and laboratory studies demonstrating $320 \mathrm{~Gb} / \mathrm{s}$ network capacity[2]. For a wide-scale commercial deployment of O-CDMA as a local-access solution, it is imperative that all required technologies be monolithically integrated onto a single photonic chip [3]. Spectral phase encoded time-spread (SPECTS) O-CDMA operates on the principle of encoding the spectral phase of a sub-picosecond pulse, resulting in an encoded pulse spread out in the time domain. The receiver reconstructs the short pulse by applying the conjugate spectral phase code to the encoded pulse. Detection of the decoded pulse is based on a nonlinear detection technique, by distinguishing between the large peak power of a properly decoded pulse and the low peak power of a spread out improperly decoded pulse. Fig. 1(a) shows the schematic of a monolithic integrated O-CDMA transceiver built by integrating discrete O-CDMA components. Previous demonstrations of arrayed waveguide (AWG) encoders and decoders, colliding pulse mode-locked lasers (CPM) and nonlinear thresholders under identical fabrication procedure proved the InP platform used in this work viable for monolithic integration [4-6]. The focus of this paper is the development of the novel, O-CDMA photonic chip compatible, $3 \mu \mathrm{m}$ deeply-etched mirrors (DEM) which form the CPM laser cavity and connects it to the O-CDMA photonic chip components.

\section{Integration technology in InP}

Integration of active and passive O-CDMA components require a common waveguide structure for optical amplification by semiconductor optical amplifiers (SOAs), optical modulation by electro-absorption (EA) modulators, and phase shifters in the AWG based encoders and decoders. The EA modulators and SOAs are formed in two growth steps. Fabrication starts with metal organic chemical vapor depositon (MOCVD) growth of the ndoped InP wafer and semiconductor optical amplifiers (SOA) layers, consisting of seven $9 \mathrm{~nm} 1.58$ Q InGaAsP quantum wells $(\mathrm{QW})$ and $5 \mathrm{~nm} 1.17 \mathrm{Q}$ InGaAsP barriers designed for maximum gain at $1550 \mathrm{~nm}$. A regrowth deposites the EA QWs and a second regrowth the p-doped cladding layers. EA, SOA and passive areas are defined by photolithography and wet etching. Dry-etching defines the waveguides. A Fe-InP layer is regrown for simultaneous passivation of the waveguide sidewalls, electrical isolation, and planarization of the surface for subsequent metallization: (AuGeTi $\backslash \mathrm{Au}$ ) on a highly doped InGaAs layer. Fig. 1(f) shows a typical waveguide of $3 \mu \mathrm{m}$ width and $0.5 \mu \mathrm{m}$ height. Refractive indices of the core and cladding are 3.3102 and 3.4511 , respectively.

Integration of the CPM is challenging due to the large bandwidth of operation and its sensitivity to reflections. Fig. 1(b) shows the CPM cavity formed symmetrically by sandwiching a $50 \mu \mathrm{m}$ absorber and two $1000 \mu \mathrm{m}$ gain sections between two DEMs. Passive waveguides between the gain section and the DEM extend the cavity to $8600 \mu \mathrm{m}$ allowing for $10 \mathrm{GHz}$ operation. All interfaces between the active and passive regions are angled at 45 degrees and facets are anti-reflection coated (AR) to reduce reflections that will adversely affect modelocking. Additionally, all components making up the CPM must have enough spectral bandwidth to accommodate the AWG based encoders and decoders $(20 \mathrm{~nm}$ ). Although the direct approach of Fresnel reflection (FR) between InP and air (30\% reflectivity) satisfies the broad spectral bandwidth requirements, it is inadequate for coupling to integrated devices on the same chip. Bandwidth constraint also eliminates the use of low-index contrast gratings which have been demonstrated in a monolithically integrated wavelength converter in InP [7]. The DEMs keep the light on-chip 


\section{JWA47.pdf}

and provide broad bandwidth reflectivity. This approach is based on photonic crystals $(\mathrm{PhC})$ which use the high index contrast between air and semiconductor to create $\mathrm{PhC}$ reflection bandgaps for optical waves[8]. Here, PhCs are unnecessary, because with a single 1-D slot the DEM, can achieve $70 \%$ reflectivity while reducing excess optical losses through multiple periods of $\mathrm{PhC}$ with not-perfectly-vertical etched walls when etched $3 \mu \mathrm{m}$ deep.
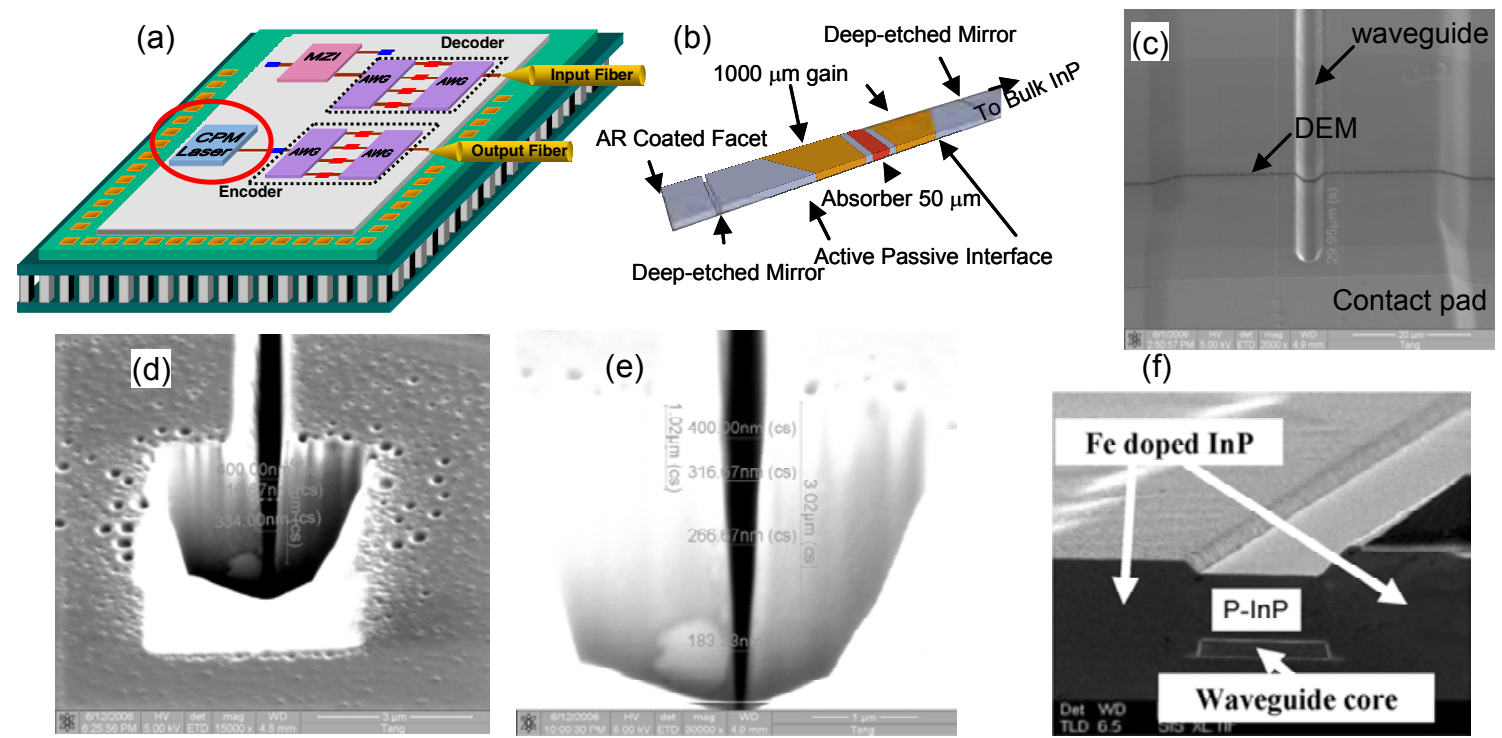

(f)

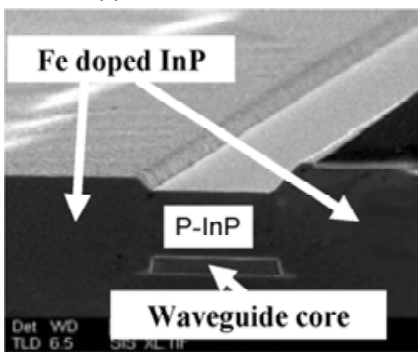

Fig. 1. (a) Integration of CPM laser with O-CDMA transceiver. (b) CPM laser structure with slotted mirrors (c) the waveguide and deep-etched mirror. (d,e) Side view of deep-etched mirror. (f) Waveguide cross section.

Fig. 1(c,d,e) show SEM images of the DEM formed using a focused ion beam (FIB) and an inductively coupled plasma-reactive ion etch (ICP-RIE) machine. The DEMs consist of a single air slot with width of $387 \mathrm{~nm}$ (1 quarter of $1550 \mathrm{~nm}$ ) etched perpendicular to an InP waveguide. Finite-difference time-domain (FDTD) simulations are used to study the reflectivity vs. wavelength and to investigate the factors that cause deviations from the ideal reflectivity $(70 \%)$. Fig. 2 (a) shows the simulated reflectivity and transmission vs. the air gap width of a slab waveguide of $0.5 \mu \mathrm{m}$. Maximum reflectivity of $57 \%$ was calculated for a $450 \mathrm{~nm}$ gap rather than the ideal case (387 $\mathrm{nm}$ gap) because of divergence of the optical mode in the gap. As the gap becomes larger, the transmission (blue) and the power remaining in the waveguide (red) decreases due to mode mismatch of the diffracting wavefront in the air gap and the waveguide. At larger gaps, the oscillations of reflection vs. gap width vanish and the reflectivity converges towards the facet reflectivity (black line). DEM reflectivity is larger than FR even for variations of the gap size up to $400 \mathrm{~nm}$. Fig. 2 (b) shows the simulated bandwidth of the DEM. The reflectivity of the DEM is essentially constant across the operation bandwidth of the CPM $(20 \mathrm{~nm}$ around $1550 \mathrm{~nm})$. Deviations of the DEM sidewalls from vertical cause the largest reduction of reflectivity due to increased scattering. According to FDTD simulations, at 14 degrees off vertical, the scattering loss reduces the reflectivity by 50\%. Fig. 2 (c) shows the measured time domain impulse response of the reflection of a DEM looking into the AR-coated facet, using an optical-vector network analyzer (OVNA). Varying coupling loss between the lensed fiber and the waveguide make exact measurements of the mirror reflectivity difficult. Including an estimation of the coupling loss between the lensed fiber and waveguide (4-6dB per pass), allows for estimation of the reflectivity to be greater than $-10 \mathrm{~dB}$.
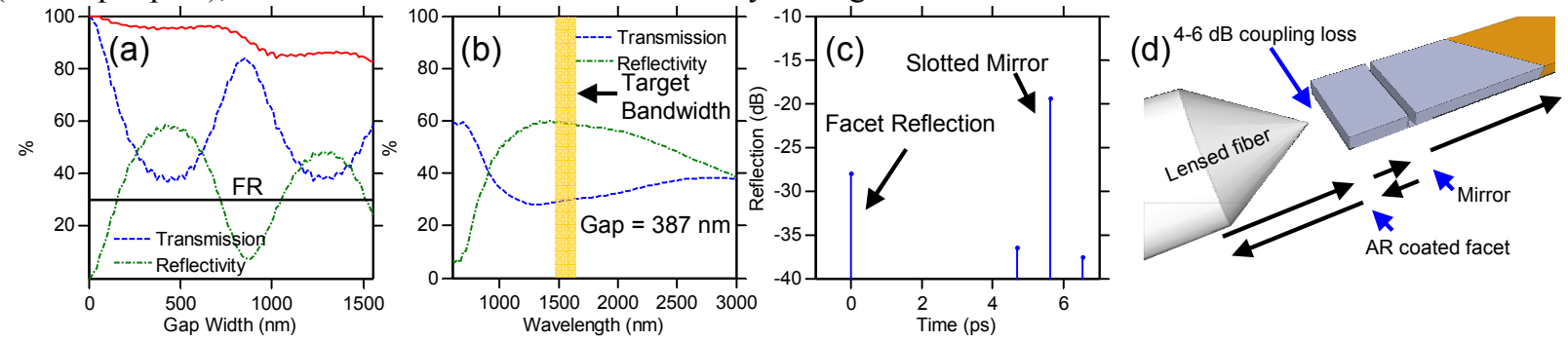

Fig. 2. FDTD simulation of (a) DEM mirror reflectivity vs. gap width at $1550 \mathrm{~nm}$ (b) DEM mirror reflectivity and transmission vs. wavelength (c) time domain measurement of mirror (d) Description of reflections.

\section{Results and discussion}

Fig. 3(a) shows the experimental setup to measure continuous wave (CW) and mode-locked operation of the CPM. Comparison of the $\mathrm{CW}$ power output vs. injection current of several lasers with and without DEMs is shown in 


\section{JWA47.pdf}

Fig. 3(b). Lasing threshold occurs at $57 \mathrm{~mA}$ only for the laser with the DEMs, indicating working DEMs. Average threshold currents for a similar CPM using FR instead are $100 \mathrm{~mA}$. Comparing the relatively low threshold current between the lasers with DEMs to FR indicates that reflectivity of the DEM is greater than the FR reflectivity $(30 \%)$.
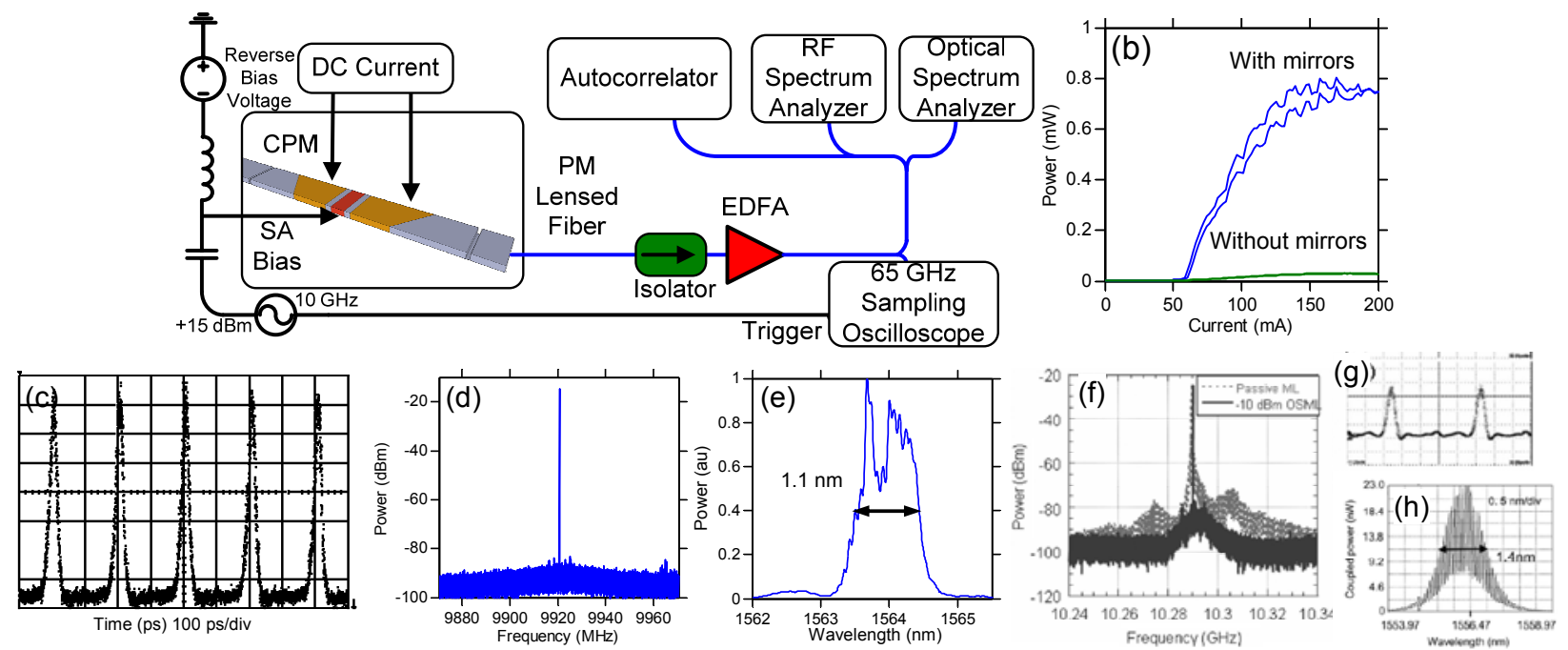

Fig. 3. (a) Measurement setup for CPM testing. (b) Power vs. continuous current laser curves. (c) sampling oscilloscope trace, (d) RF spectrum, (e) Optical spectrum of hybrid-modelocked CPM formed by DEM. (f) RF spectrum, (g) sampling oscilloscope trace (h) optical spectrum of hybrid-modelocked CPM formed by FR.

A $10 \mathrm{GHz}$ RF drive signal from a microwave synthesizer in combination with a reverse voltage DC bias to the absorber, synchronizes the CPM to an external reference. A comparison of the hybrid modelocking characteristics of a CPM formed by DEM (DEM-CPM) as shown in Fig. 3(c,d,e) and a CPM formed by facets (FR-CPM) as shown in Fig. 3(f,g,h) demonstrates the DEM effectiveness for integration in the O-CDMA platform. Both the DEM-CPM and FR-CPM produce a stable, 100 ps period pulse train indicated by both the narrow spike in the RF spectra and sampling oscilloscope traces. The timing jitter is estimated by integrating the single side band (SSB) phase noise spectrum offset $20 \mathrm{kHz}$ and $80 \mathrm{MHz}$ to be $243 \mathrm{fs}$ for the DEM-CPM compared to 283 fs the FR-CPM. Although both CPMs show stable mode-locking, the optical modes of the DEM-CPM ouput are not as clearly distinguished as the FR-CPM output and the pulse width is larger (10 ps compared to $1.8 \mathrm{ps})$. The instability is believed to be caused by the unwanted but weak reflection from the AR coated facet. Strong evidence supporting this claim is the ripple in the optical spectrum of the DEM-CPM output corresponding to the Fabry-Perot cavity formed by the AR coated facet and the DEM. On a future integrated O-CDMA transceiver designs, facets will be angled to reduce reflections.

\section{Conclusion}

We demonstrated integration of a CPM onto the O-CDMA photonic chip platform using $3 \mu \mathrm{m}$ deep etched slotted mirrors. Performance of the CPM with DEM is comparable to a CPM using etched facets.

\section{References}

[1] X. Wang, N. Wada, T. Miyazaki, G. Cincotti, and K. Kitayama, "Field trial of 3-WDM $\times 10-O C D M A \times 10.71$ gbps, trulyasynchronous, WDM/DPSK-OCDMA using hybrid E/D without FEC and optical threshold," in OFC 2006 (OSA, 2006).

[2] W. Cong, C. Yang, R. P. Scott, V. J. Hernandez, N. K. Fontaine, B. H. Kolner, J. P. Heritage, and S. J. B. Yoo, "Demonstration of 160- and 320-Gb/s SPECTS O-CDMA network testbeds," IEEE Photonics Technologies Letters, 18, 1567-1569 (2006).

[3] R. G. Broeke, J. Cao, C. Ji, S.-W. Seo, Y. Du, N. K. Fontaine, J.-H. Baek, J. Yan, F. M. Soares, F. Olsson, S. Lourdudoss, A.-V. Pham, M. Shearn, A. Scherer, and S. J. B. Yoo, "Optical-CDMA in InP," Journal of Selected Topics in Quantum Electronics, 13 (2007).

[4] J. Cao, R. G. Broeke, N. Fontaine, W. Cong, C. Ji, Y. Du, N. Chubun, K. Aihara, A.-V. Pham, J. P. Heritage, B. H. Kolner, S. J. B Yoo, F. Olsson, S. Lourdudoss, and P. L. Stephan, "Error-free spectral encoding and decoding operation of InP O-CDMA encoder," in OFC 2006 (OSA, 2006), Paper OWL2.

[5] F. M. Soares, N. K. Fontaine, Jiangwei, S. Seo, J. Baek, R. G. Broeke, J. Cao, K. Okamoto, and S. J. B. Yoo, "Compact InP-Based 16Channel O-CDMA Encoder/Decoder," in LEOS 2007 (OSA, 2007).

[6] C. Ji, R. G. Broeke, Y. Du, C. Jing, N. Chubun, P. Bjeletich, F. Olsson, S. Lourdudoss, R. Welty, C. Reinhardt, P. L. Stephan, and S. J. B. Yoo, "Monolithically integrated InP-based photonic chip development for O-CDMA systems," IEEE Journal of Selected Topics in Quantum Electronics, 11, 66-77 (2005).

[7] V. Lal, M. L. Masanovic, J. A. Summers, L. A. Coldren, and D. J. Blumenthal, "Performance optimization of an InP-based widely tunable all-optical wavelength converter operating at $40 \mathrm{~Gb} / \mathrm{s}$," IEEE Photonics Technology Letters, 18, 577-579 (2006).

[8] E. Yablonovitch, T. J. Gmitter, and K. M. Leung, "Photonic Band Structure: The Face-Centered-Cubic Case Employing Nonspherical Atoms," Physics Review Letters, 67, 2295-2299 (1991).

This work was supported in part by DARPA and SPAWAR under agreement number N66001-02-18937 and by the AFOSR through the UC Davis Center for Digital Security. 